\title{
Evaluation of Anti-Retroviral Combination Therapy In Patients With HIV/Aids Injecting Drug Users
}

\author{
Ahmad Riyadi $^{1,2}$, Arif Wardoyo ${ }^{2}$ and Syamsudin ${ }^{3 *}$ \\ ${ }^{I}$ Master of Pharmaceutical Sciences, Faculty of Pharmacy, Pancasila University \\ Jl. Srengseng Sawah, Jagakarsa, South Jakarta, Indonesia \\ ${ }^{2}$ Drug Dependence Hospital, Jalan Lapangan Tembak No. 75 Cibubur Jakarta Timur 13720 \\ ${ }^{3}$ Faculty of Pharmacy, Pancasila University, Jakarta, Indonesia
}

\begin{abstract}
HIV stands for 'Human Immunodeficiency Virus'. HIV is a retrovirus that infects cells of the human immune system (mainly CD4), and destroys or impairs their function. This viral infection results in a constant decrease in human immune system and eventually results in immunodeficiency. Indonesia is one of the countries that show increasing number of HIV/AIDS cases. By June 2011, 26.483 cumulative HIV/AIDS cases were reported in Indonesia. Drug users are a major factor in the spread of HIV/AIDS infections. Antiretroviral (ARV) therapy in patients with HIV infection aims at suppressing replication in a maximum way for a long period, restoring and maintaining the body's immune. It is important to extend and improve the quality of life to reduce the morbidity and mortality. Efficacy of the combination of three of antiretroviral drugs was better than the combination of two types of antiretroviral, where there is a decrease in viral load to undetectable level and increased CD4 lymphocyte count. This study was conducted compare several combinations of antiretroviral, that is Lamivudine +Zidovudine+ Nevirapine, Lamivudine+Zidovudine +Efavirenz, Lamivudine +Stavudine + Nevirapine and Lamivudine+Stavudine+Efavirenz, in terms of effectiveness, side effects, adherence and antiretroviral drug costs in patients HIV/AIDS drug users in Drug Dependence Hospital Jakarta. The study was conducted in a retrospective way, using data obtained from medical records of patients from January 2005 to December 2010 in patients with CD4 $\leq 350 \mathrm{cells} / \mathrm{mm}^{3}$ and a minimum of 6-12 months of antiretroviral treatment. Of the 333 patients, 100 patients had antiretroviral treatment and met the inclusion criteria. The results showed effectiveness of combination antiretroviral therapy in that there was an increase in the number of CD4; the increase was found to be different in each antiretroviral combination. However, statistical test with ANOVA showed that the increases in CD4 had no significant difference. Nausea is a common side effect in HIV/AIDS patients who received antiretroviral therapy. Of the 100 patients, $94 \%$ adhered to the therapy and the combination of Lamivudine+Zidovudine +Efavirenz required the highest cost, compared to the other combinations.
\end{abstract}

KEYWORDS : HIV/AIDS, CD4 count, antiretroviral combination

\section{INTRODUCTION}

HIV stands for 'Human Immunodeficiency Virus'. HIV is a retrovirus that infects human immune system cells (particularly CD4) and then destroys or disrupts their functions. The viral infection results in a constant decline in human immune system and eventually results in immunodeficiency. ${ }^{(1)}$ Indonesia is one of the countries that show increasing number of HIV/AIDS cases. HIV/AIDS case was firstly identified in Bali in 1987. By June 2011, 26.483 cumulative HIV/AIDS cases were reported in Indonesia. Based on the infection mode to the Injecting Drug User (IDU), cumulative percent of HIV/AIDS cases in Indonesia was 36,2\%.(2)

Therapy for HIV/AIDS patients mush be well planned. Before the therapy is initiated, the patients must receive counseling session on the benefits of therapy and possible side effects. Then, the therapy must be followed by monitoring for the treatment success. ${ }^{(3)}$ To initiate antiretroviral therapy, CD4 must be counted. Beginning the ARV therapy on adult patients with CD4 count $<350$ was found to be effective in terms of both cost and clinical benefits. ${ }^{(4)}$ Antiviral (ARV) therapy with three or more combinations could dramatically reduce the mortality rate and AIDS-related morbidity. Even though not a healing solution, combined ARV therapy lengthens the life of HIV-positive patients, makes them healthier, and enables them to live more productively by increasing the CD4 counts. To make antiretroviral treatment effective for a long period of time, different ARV medications must be combined; this is called Highly Active Anti-Retroviral Therapy' (HAART). When a single medication is used, within a certain period of time, drug resistance may develop because of viral changes. When this is the case, the medication will not be effective anymore and the viruses in the same number will be produced again. ${ }^{(5)}$ Antiretroviral treatment guidelines for HIV infection in adults and adolescents recommend the use of two Nucleoside Reverse Transcriptase Inhibitors (NRTIs) in addition to a single Non-nucleoside Reverse Transcriptase Inhibitor (NNRTI). Right now, there are two NNRTIs, namely Nevirapine or Effavirenz, both of 
which show the same effectiveness as that of antiviral therapy. ${ }^{(4)}$ Efficacy of three combined ARVs was found to be better than the combined two ARVs, since a decrease in viral load up to an undetectable level and increase in the number of lymphocyte DC4 counts were found. ${ }^{(6)} \mathrm{HIV}$ therapy using combined three antiretroviral medications was found to be very effective in terms of cost. ${ }^{(7)}$ Antiretroviral regimens with two NRTI and PI, NNRTI or NRTI showed comparable effectiveness. The principle of simplicity, such as the daily doses (number of tablets for a day), must be a consideration in selecting a treatment regimen. ${ }^{(6)}$ The study aims at answering some questions related to the comparison of CD4 count, body weight, adverse effects, adherence, and treatment cost of some combined ARV therapies for HIV/AIDS patients using narcotics, psychotropic, and addictive substances at Drug Dependence Hospital, Jakarta.

\section{MATERIALS AND METHODS}

Focus : The study was conducted at the HIV outpatient clinic of Drug Dependence Hospital, Jakarta - a specialty hospital that provides services to those with abuse of drugs, alcohol and other addictive substances.

Population : Participants of the research were all new admissions for HIV/AIDS cases at Drug Dependence Hospital Jakarta from January 2005 to December 2010; 100 patients were selected.

Study design : This was a retrospective study. Random sampling was done every day during routine visits until the desired sample size was reached. Samples of the research were all new admissions for HIV/AIDS cases at Drug Dependence Hospital Jakarta from January 2005 to December 2010; 300 patients were admitted during the period. The criterion for statistical significance was $\mathrm{p}$ value $<0.05$.

Inclusion criteria : HIV-1 infected male and female patients aged 15 years and older on HAART, regularly reviewed for complaints during six months or more of treatment were deemed eligible for this study. Patients were Injecting Drug Users (IDU) with CD4 $\leq 350 \mathrm{cells} / \mathrm{mm}^{3}$, under ARV treatment for $6-12$ months, and having one of the following ARV therapies: Combination I: Lamivudine + Zidovudine + Nevirapine; Combination II: Lamivudine + Zidovudine + Efavirenz; Combination III: Lamivudine + Stavudine + nevirapine; Combination IV Lamivudine + Stavudine + Efavirenz.

Exclusion criteria : Patients on HAART for less than 6 months

Patient assessment : The Comprehensive Care Centre operated five days a week. All patients underwent full evaluation at initial and subsequent follow-up visits. Data on patient characteristics such as age, gender, marital status, occupation, level of education, WHO clinical staging, current and prior anti-retroviral therapy, physical examination findings and baseline CD4 counts were recorded in the patients' charts.

\section{RESULTS}

The Drug Dependence Hospital (RSKO) Jakarta is one of referral hospitals for HIV/AIDS therapy. It provides healthcare services and management for HIV/AIDS patients. The team of doctor works by referring to National Guidelines for ARV Therapy issued by the central government and by referring to WHO guidelines. Patients' data for the research were taken from January 2005 - December 2010. Of the 333 patients recorded to have ARV therapy, 100 patients met the inclusion criteria.

Patients' baseline characteristics

The Patients in this research were mostly male (99\%), within age range of 25-49 years (82\%), living in East Jakarta (37\%), unmarried (65\%), unemployed (77\%), and Senior High School graduates (74\%).

Table 1. Demographic characteristic of the study population

\begin{tabular}{llcc}
\hline & \multicolumn{1}{c}{ Variable } & No of patients & Mean (\%) \\
\hline Age & $15-19$ & 0 & 0 \\
& $20-24$ & 18 & 18 \\
\multirow{4}{*}{ Gender } & $25-49$ & 82 & 82 \\
& $>50$ & 0 & 0 \\
Marital Status & Male & 99 & 99 \\
& Female & 1 & 1 \\
Employment & Unmarried & 65 & 65 \\
& Married & 35 & 35 \\
& Employed & 23 & 23 \\
\hline
\end{tabular}




\begin{tabular}{llcc}
\hline status & Unemployed & 77 & 77 \\
Education & Elementary School & 1 & 1 \\
& Junior High School & 4 & 4 \\
& Senior High School & 74 & 74 \\
& Bachelor & 21 & 21 \\
\end{tabular}

The greatest proportion of HIV infections found at Drug Dependence Hospital occurred with shared Injecting drug use (IDU).

ARV Combination : In this research, the mostly used ARVs were Combination I (lamivudine + zidovudine) + nevirapine (55\%). The use of Combination I was the first-line ARV combination specified by the government for the patients without liver function and blood disorders. The Combination I was chosen since it was easily available and produced in Indonesia.

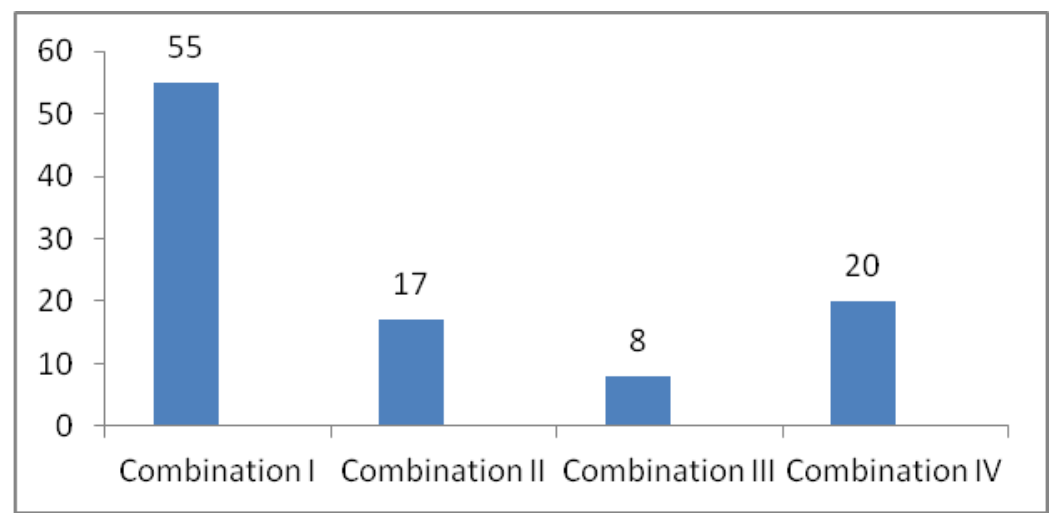

Figure 1. ARV Combination used for Therapy in HIV/AIDS Patients

Initial Diagnosis : An investigation for the initial diagnosis of HIV/AIDS patients may provide early diagnosis that the patients will get the treatment earlier.

Table II. Initial Diagnoses

\begin{tabular}{lcc}
\hline \multicolumn{1}{c}{ Initial Diagnosis } & \multicolumn{2}{c}{$\mathbf{n = 1 0 0}$} \\
\cline { 2 - 3 } & $\begin{array}{c}\text { No. of } \\
\text { Patients }\end{array}$ & $\begin{array}{c}\text { Mean } \\
(\%)\end{array}$ \\
\hline $\begin{array}{l}\text { Fever, Decreasing BW, } \\
\text { weakness, nausea }\end{array}$ & 29 & 27.10 \\
\hline $\begin{array}{l}\text { Fever, nausea, vomiting, } \\
\text { asphyxia, cough }\end{array}$ & 30 & 28.04 \\
\hline $\begin{array}{l}\text { Fever, diarrhea, dry mouth, } \\
\text { candidiasis }\end{array}$ & 18 & 16.82 \\
\hline $\begin{array}{l}\text { Fever decreasing BW, cough, } \\
\text { Candidiasis }\end{array}$ & 30 & 28.04 \\
\hline
\end{tabular}

Adherence : Adherence to therapy refers to a condition in which a patient willingly adheres to the treatment regimen, rather than a mere submission to the health staff's order. Willingness is very important to ensure that the patient adheres to the dosing time. The adherence must be regularly monitored and evaluated at each visit. Failure of ARV therapy was frequently attributed to non-adherence of the patient to take ARV doses. In this research, adherence was assessed based on the attendance for doctor counseling and timeliness in each visit. Percent visit adherence was calculated each month by counting the difference in the visiting date in the next month. Most HIV/AIDS patients (94\%) at Drug Dependence Hospital, Jakarta, adhered to the ARV therapy. 
Opportunistic Infection : The course of HIV infection is relatively long - up to 10 years. As a result, a HIVinfected patient may not recognize his condition. HIV infection is usually detected when there is an opportunistic infection that leads to HIV infection diagnosis. The opportunistic infections include oral candidiasis and tuberculosis. The types of opportunistic infections among HIV/AIDS patients include oral candidiasis, lung TB, cerebral toxoplasmosis, chronic diarrhea, peritoneal tuberculosis, Tuberculous lymphadenitis, and tuberculous meningitis. In patients with low CD4 count, some opportunistic infections may occur at the same time. : In this research, the most common opportunistic infections were oral candidiasis (40\%) and lung TB (24\%), since both oral candidiasis and TB may infect patients with high CD4 count. Therefore, even though a patient has ARV therapy and CD4 increases, oral candidiasis may recur during the ARV therapy. According to opportunistic infection data reported by June 2011, TBC, diarrhea, and candidiasis were the most common cases. $^{(8)}$

Adverse effects : Side effect is one of the important aspects in ARV therapy. Moreover, side effect is frequently used as a medical reason to substitute and/or to terminate ARV therapy. The patients sometimes cease the therapy on their own initiatives due to the adverse effects. Drug adverse effects must not be a barrier to initiate ARV therapy, since not all patients develop adverse effects and most drug adverse effects could be managed well. This is far more beneficial compared to the death risk when the patients do not receive ARV therapy.(9) In this research, 34\% of the patients did not report any adverse effects. One of the common adverse effects among HIV/AIDS patients was nausea (30\%), since ARV makes use of peroral pathway, where it may irritate and increase gastric acid level.

Table III. Drug Adverse Effects Based on ARV Combination

\begin{tabular}{clcc}
\hline ARV & \multicolumn{1}{c}{ Adverse Effects } & Freq. & Percent \\
\hline & Nausea & 16 & 29.09 \\
& Anemia & 6 & 10.91 \\
& Skin rush & 4 & 7.27 \\
\hline Combination I & Headache & 6 & 10.91 \\
& Increasing AST/ALT & 2 & 3.64 \\
& Itching & 3 & 5.45 \\
& No adverse effect & 18 & 32.73 \\
\hline \multirow{3}{*}{ Combination II } & Nausea & 3 & 17.65 \\
& Anemia & 2 & 11.76 \\
& Headache & 5 & 29.41 \\
& No adverse effect & 7 & 41.18 \\
\hline & Nausea & 1 & 12.50 \\
& Skin rush & 1 & 12.50 \\
Combination III & Increasing AST/ALT & 1 & 12.50 \\
& Itching & 1 & 12.50 \\
& No adverse effect & 4 & 50.00 \\
& Nausea & 10 & 50.00 \\
& Anemia & 3 & 15.00 \\
\hline Combination IV & Headache & 1 & 5.00 \\
& Itching & 1 & 5.00 \\
& No adverse effect & 5 & 25.00 \\
\hline
\end{tabular}

Increase in CD4 Count in the Four Combinations :In this research, measurement of CD4 count in 100 patients that met the inclusion criteria revealed an increase in the CD4 count after 6-12 months of ARV therapy. The National Guideline for ARV Therapy recommends CD4 measurement for every 6 months to determine the effects and effectiveness of each ARV combination therapy. The four ARV combinations used in this research showed better effectiveness since they provide a CD4 increase of $\geq 50$ cells $/ \mathrm{mm}^{3}$ within $6-12$ months of therapy. Table IV. Average increase in CD4 count related to each ARV Combination Therapy

\begin{tabular}{|c|c|c|c|}
\hline ARV Combination & $\begin{array}{c}\text { No. of } \\
\text { Patients }\end{array}$ & $\begin{array}{c}\text { Mean } \\
\text { dCD4 }\end{array}$ & SD \\
\hline I & 55 & 109,02 & 75,75 \\
\hline II & 17 & 117,65 & 75,33 \\
\hline III & 8 & 80,38 & 59,49 \\
\hline IV & 20 & 130,45 & 80,40 \\
\hline
\end{tabular}


Comparison of Body Weight Increase Based on the ARV Combinations :The research also monitored the patient's body weight at the beginning of therapy and during the 6-12 months of therapy.

Table V. Average Increase of Bodyweight for each ARV combination

\begin{tabular}{|c|c|c|c|}
\hline ARV Combination & No. & $\begin{array}{c}\text { Mean } \\
\text { dCD4 }\end{array}$ & SD \\
\hline I & 55 & 5,7818 & 9,689 \\
\hline II & 17 & 2,4118 & 6,3152 \\
\hline III & 8 & 3,25 & 3,8452 \\
\hline IV & 20 & 4,1 & 6,9578 \\
\hline
\end{tabular}

Of the 100 patients that met inclusion criteria, 55 patients received Combination I (AZT+3TC+NVP) with average/mean body weight increase of $5,7818 \mathrm{~kg}, 17$ patients received Combination II (AZT+3TC+EFV) with average/mean body weight increase of $2,4118 \mathrm{~kg}, 8$ patients received Combination III (d4T+3TC+NVP) with average/mean body weight increase of $3,25 \mathrm{~kg}$, and 20 patients received Combination IV (d4T+3TC+EFV) with average/mean body weight increase of $4,1 \mathrm{~kg}$.

\section{DISCUSSION}

In this research, the mostly used ARVs were Combination I (lamivudine + zidovudine) + nevirapine $(55 \%)$. The use of Combination I was the first-line ARV combination specified by the government for the patients without liver function and blood disorders. The Combination I was chosen since it was easily available and produced in Indonesia. Other studies compared ARV combination in terms of virologic and immunological responses. They included a study by $\mathrm{Li}$ in China that assessed virologic response. He showed that virologic response was almost similar between the combination of zidovudin and stavudine. Virologic response in a prospective study on 198 patients with HAART in 52 weeks of examination showed a plasma viral load of $<50$ copies/patient in Group B that received NVP+3TC+d4T and those in Group C that received NVP+AZT+3TC, compared to those in Group A that received NVP+AZT+ddl [68,2\%, 69\% vs 39,7\%, respectively, $p<0,001$ ) $(\mathrm{Li}, 2008)$. Another retrospective study in Uganda showed a significant efficacy of AZT+3TC+EFV combination in 31 weeks of examination, with an average increase in CD4+ lymphocyte of 183. Virologic (viral load) suppression was not detected in $88,9 \%$ patients examined 11,6 weeks after the therapy.

Of the 100 patients that met inclusion criteria, 55 patients received Combination I (AZT+3TC+NVP) with average/mean CD4 increase of 109,02 cells $/ \mathrm{mm}^{3} ; 17$ patients received Combination II II (AZT+3TC+EFV) with average/mean CD4 increase of 117,65 cells $/ \mathrm{mm}^{3}, 8$ patients received Combination III (d4T+3TC+NVP) with average/mean CD4 increase of $80,38 \mathrm{cells} / \mathrm{mm}^{3}$, and 20 patients received Combination IV $(\mathrm{d} 4 \mathrm{~T}+3 \mathrm{TC}+\mathrm{EFV})$ with average/mean CD4 increase of $130,45 \mathrm{cells} / \mathrm{mm}^{3}$. Some previous studies suggested that baseline CD4 determined the increase of CD4 in the patients. The higher the CD4 count of HIV/AIDS patients when initiating the treatment, the higher the increase in CD4 counts (Evans, 2007). Patients that initiated the therapy with CD4 count of less than 200 cells $/ \mathrm{mm}^{3}$ showed almost two-fold failure of treatment $(\mathrm{HR}: 1,9)$ compared to those who initiated the therapy with CD4 count of more than 200 cells $/ \mathrm{mm}^{3}$ (Robbin, 2007). An adequate response in the patients receiving ARV therapy was defined as an annual CD4 increase of 50-150 cells $/ \mathrm{mm}^{3}$, with a quick response usually seen during the first month of treatment. ${ }^{(9)}$ Increasing body weight among the HIV/AIDS patients represents a clinical response on the ARV therapy. Increasing CD4 counts among the HIV/AIDS patients is attributable to the improvement of immune system. The immune system does not always respond, so does the increase of body weight. This can be used as a marker of clinical condition. ${ }^{(10)}$ Comparison of body weight increase based on the ARV combination using ANOVA revealed a significance value of 0,474 (higher than 0,05 ). This means that the increase of body weight for the four ARV combination therapies was not statistically different.

Increase in the CD4 count among HIV/AIDS patients were the result of immune system improvement. The body does not always respond to the increase of body weight, which can be used as a clinical sign. ${ }^{(10)}$ ANOVA showed a significance value of 0,474 - (above 0.05 ); therefore, increase in the body weight related to the four ARV combinations was not statistically different. The combination of the non-nucleoside reverse transcriptase inhibitor nevirapine (NVP), and two nucleoside reverse transcriptase inhibitors, stavudine (d4T) or zidovudine (AZT) and lamivudine (3TC), is widely used as first-line therapy (the second strategy), especially in low-resource countries. 


\section{CONCLUSION}

Of the four ARV combinations used in HIV/AIDS patient therapy, all were found to be effective in increasing CD4 counts. Statistical analyses did not reveal any significant difference in the increase of CD4 count and the increase of body weight. Combination I (Lamivudine + Zidovudine + Nevirapine ) was the most commonly used combination of all. The most common side effect of ARV therapy was nausea. Adherence of HIV/AIDS patients to ARV therapy at RSKO was $94 \%$.

\section{REFERENCES}

[1] Shete, A (2013). Current Trends in HIV/AIDS. J HIV AIDS Infect Dis 1: 1-2

[2] Ibrahim K, Songwathana P, Boonyasopun U, Francis K (2010) The HIV/AIDS epidemic in Indonesia: does primary health care as a prevention and intervention strategy work? Int J Nurs Pract 16: 87-91.

[3] Robertson J, Meier, M, Wall J, Ying J, Fichtenbaum C (2006). Immune reconstitution syndrome in HIV: validating a case definition and identifying clinical predictors in persons initiating antiretroviral therapy. Clin Infect Dis;42:1639-46.

[4] Costello C, Nelson K, et al (2005). Predictors of low CD4 count in resource-limited settings based on an antiretroviral-naive heterosexual Thai population. JAIDS;39:242-8.

[5] Alvarez (2004). Tenovir and Zidovudine/Lamivudine as Triple Therapy for Infection, Drexer University College of Medicine, Philadelphia, PA, USA, International Journal of Infection Disease, ICID Vol. 8

[6] Kenneth A. Freedberg, M.D., et al. (2001). The Cost Effectivennes Of Combination Antiretroviral Theraphy For HIV Disease, The New England Journal of Medicine, vol. 344, No.11

[7] Koening SP et al, (2011) Cost Effectiveness of early versus antiretroviral therapy in HIV-infected adults in Haiti. PLoS Med 8(9).

[8] Sungkanuparph S, Kiertiburanakul S, et al (2005). Initiation of highly active antiretroviral therapy in advanced AIDS with CD4 $<50$ cells/mm3 in a resource-limited setting: efficacy and tolerability. International Journal of STD \& AIDS;16:243-6.

[9] WHO: Guidelines for using HIV testing technologies in Surveillance: selection, evaluation and implementation. www.whoint/hiv/pub/surveillance

[10] Evans, R and Becker, S (2009). Children caring for parents with HIV and AIDs: Global issues and Policy responses. Bristol: The Policy Press 